\title{
HK2 is associated with the Warburg effect and proliferation in liver cancer: Targets for effective therapy with glycyrrhizin
}

\author{
ZENGPENG SUN, ZHIGUO TAN, CHUANG PENG and WEIMIN YI \\ Department of Hepatobiliary Surgery, The First Affiliated Hospital of Hunan Normal University \\ (Hunan Provincial People's Hospital), Changsha, Hunan 410005, P.R. China
}

Received July 23, 2020; Accepted January 15, 2021

DOI: $10.3892 / \mathrm{mmr} .2021 .11982$

\begin{abstract}
Glycyrrhizin (GA) is the most essential active ingredient in licorice root, and has a wide range of biological and pharmacological activities. The present study aimed to conduct a detailed analysis of the effects of GA on liver cancer (LC) cell proliferation and the Warburg effect. Hexokinase-2 (HK2) is a glycolytic enzyme that catalyzes the Warburg effect. To this end, the LC HepG2 cell line was transfected with small interfering RNA-HK2 or pCDNA3.1-HK2, followed by GA treatment. A Cell Counting Kit-8 assay and EdU staining were employed to evaluate the proliferation rate of LC cells. The expression levels of HK2 and the phosphorylation level of AKT were measured by reverse transcription-quantitative PCR and western blotting, respectively. Furthermore, the glucose uptake capacity and lactic acid content were assessed by kits, and the glycolysis level was evaluated by assessing the extracellular acidification rate (ECAR) and the oxygen consumption rate (OCR). A pronounced increase in the OCR, and decreases in the cell proliferation, glucose uptake capacity, lactic acid content, ECAR and HK2 expression were detected in LC cells subjected to GA treatment or HK2-knockdown. Conversely, overexpression of HK2 reversed these trends, indicating that glycyrrhizin may inhibit LC cell proliferation and the Warburg effect through suppression of HK2. In addition, it was revealed that the PI3K/AKT signaling pathway was associated with LC cell proliferation and the Warburg effect; notably, treatment of LC cells with the AKT agonist SC79 induced elevation of the ECAR, cell proliferation, glucose uptake capacity, lactic acid content, phosphorylated-AKT and HK2 expression, and suppressed the OCR. In conclusion, GA may inhibit the Warburg effect and cell proliferation in LC by suppressing HK2 through blockade of the PI3K/AKT signaling pathway.
\end{abstract}

Correspondence to: Dr Chuang Peng, Department of Hepatobiliary Surgery, The First Affiliated Hospital of Hunan Normal University (Hunan Provincial People's Hospital), 61 West Jiefang Road, Furong, Changsha, Hunan 410005, P.R. China

E-mail: pengchuang1966@163.com

Key words: glycyrrhizin, Warburg effect, cell proliferation, PI3K/AKT, hexokinase 2, liver cancer HepG2 cell line

\section{Introduction}

Hepatocellular carcinoma is responsible for $>90 \%$ of liver cancer (LC) cases and is the second most common cause of cancer-associated death worldwide $(1,2)$. The main risk factors for LC include viral infection, excessive alcohol consumption, non-alcoholic fatty liver disease and toxins, such as aflatoxin-B found in contaminated food $(3,4)$. Unfortunately, most patients are diagnosed at a late stage, given the asymptomatic nature of LC, and advanced LC is not amenable to curative strategies (5). Therefore, identifying alternative and innovative therapeutic strategies for the systematic treatment of LC is important.

Altered energy metabolism has been accepted as one of the typical hallmarks of cancercells (5). The Warburg effect is defined as a phenomenon in which glucose is preferentially utilized by glycolysis, rather than by oxidative phosphorylation, and is characterized by high rates of lactate production and glucose uptake even in the presence of oxygen (6,7). Phosphofructokinase-1, pyruvate kinase M2, pyruvate dehydrogenase kinase-1, lactate dehydrogenase and hexokinase-2 (HK2) have emerged as indispensable glycolytic enzymes that catalyze the Warburg effect (8). Among these glycolytic enzymes, HKs catalyze the irreversible first step of the glycolytic pathway in which glucose is phosphorylated to glucose-6-phosphate with the concomitant dephosphorylation of adenosine triphosphate (9). Notably, HK2 has been identified as a central player in the Warburg effect and has been proposed as a metabolic target for the development of cancer therapies (10). Therefore, understanding how the Warburg effect is regulated in cancer is particularly relevant for identifying novel therapeutic interventions.

Some traditional Chinese medicines have attracted increasing attention due to their potent antitumor activities and potential as candidates for the advancement of new antitumor drugs $(11,12)$. Extracted from the roots of Glycyrrhiza glabra, glycyrrhizin (GA) has been reported to possess numerous pharmacological effects, including anti-inflammatory and anti-viral activities (13). Furthermore, it has recently been demonstrated that GA has a markedly hepatoprotective effect (13-15); however, the association between GA and the regulation of cellular metabolism in LC remains largely unexplored.

The aim of the present study was to investigate the regulatory association between GA and glycolytic enzyme HK2 in order to improve the current understanding of the mechanisms underlying LC progression. 


\section{Materials and methods}

Short tandem repeat (STR) analysis of the LC HepG2 cell line. DNA was extracted using a genome extraction kit (Axygen; Corning, Inc.) and amplified by the 21-STR amplification protocol. The STR locus and amelogenin gene were examined on an ABI 3730XL DNA analyzer (Applied Biosystems; Thermo Fisher Scientific, Inc.).

Cell culture. The LC HepG2 cell line was obtained from The Cell Bank of Type Culture Collection of The Chinese Academy of Sciences. Cells were cultured in Dulbecco's modified Eagle's medium (DMEM; Gibco; Thermo Fisher Scientific, Inc.) supplemented with $10 \%$ fetal bovine serum (FBS; Gibco; Thermo Fisher Scientific, Inc.) in an atmosphere containing $5 \% \mathrm{CO}_{2}$ at $37^{\circ} \mathrm{C}$. Small interfering (si)-HK2 (ACGACAGCATCATTGTTAA), si-negative control (NC), pCDNA3.1-HK2 vector and pCDNA3.1 vector were synthesized by Shanghai GenePharma Co., Ltd. The cells were seeded in 6-well plates at a density of $3 \times 10^{5}$ cells/well and underwent cell transfection once cell confluence reached $40-60 \%$. Cell transfection was conducted using Lipofectamine ${ }^{\circledR} 2000$ (Invitrogen; Thermo Fisher Scientific, Inc.) according to the manufacturer's instructions. The transfection dose of si-HK2 and pcDNA3.1-HK2 was $2 \mu \mathrm{g}$ and transfection time was $24 \mathrm{~h}$. After $24 \mathrm{~h}$, the cells were assigned to the following nine groups accordingly: GA group (exposed to $100 \mu \mathrm{g} / \mathrm{ml} \mathrm{GA}$ for $24 \mathrm{~h}$; Tokyo Chemical Industry Co., Ltd.), si-HK2 group (transfected with si-HK2), si-NC group (transfected with si-NC), pCDNA3.1-HK2 group (transfected with pCDNA3.1-HK2), pCDNA3.1 group (transfected with pCDNA3.1), pCDNA3.1-HK2 + GA group (transfected with pCDNA3.1-HK2 followed by $24 \mathrm{~h}$ treatment with $100 \mu \mathrm{g} / \mathrm{ml}$ GA), SC79 group (exposed to $4 \mu \mathrm{g} / \mathrm{ml}$ AKT agonist SC79 for $6 \mathrm{~h}$; Calbiochem; Merck KgaA), SC79 + GA group (exposed to $4 \mu \mathrm{g} / \mathrm{ml} \mathrm{SC} 79$ for $6 \mathrm{~h}$ and $100 \mu \mathrm{g} / \mathrm{ml} \mathrm{GA}$ for $24 \mathrm{~h}$ ) and control group (untreated).

Cell Counting Kit-8 assay. Cells were seeded into 96-well plates (5,000 cells/well). A total of three replicates were set for each sample. LC cells HepG2 were treated with different concentrations of GA solution $(0,25,50,75,100$ and $125 \mu \mathrm{g} / \mathrm{ml})$ for $24 \mathrm{~h}$. Then, cells were analyzed daily for 4 consecutive days to assess cell proliferation by CCK-8 assay. Briefly, $10 \mu$ l CCK- 8 solution (Thermo Fisher Scientific, Inc.) was added to each well and the plates were incubated at $37^{\circ} \mathrm{C}$ for $2 \mathrm{~h}$. The absorbance of the wells was determined at a $450-\mathrm{nm}$ wavelength using a microplate absorbance reader. Cell viability $(\%)=($ average OD value of the experimental group - average OD value of the Blank group)/(average OD value of the Control group - average OD value of the Blank group) $x 100 \%$.

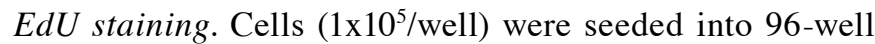
plates and $100 \mu \mathrm{l} \mathrm{EdU} \mathrm{(50} \mu \mathrm{M}$; Sigma-Aldrich; Merck KGaA) was added to each well for 2 -h incubation at $37^{\circ} \mathrm{C}$. Subsequently, cells were fixed for $30 \mathrm{~min}$ at room temperature with $50 \mu \mathrm{l}$ fixation buffer (4\% paraformaldehyde; Beyotime Institute of Biotechnology). Following removal of the fixation buffer from the plates, the cells were incubated with $50 \mu 12 \mathrm{mg} / \mathrm{ml}$ glycine at room temperature for $5 \mathrm{~min}$, prior to washing with $100 \mu 1$ PBS, exposure to $100 \mu 1$ permeabilization buffer (phosphate-buffered saline containing 0.3\% Triton X-100; Beyotime Institute of Biotechnology) and further washing with $100 \mu$ PBS. The cells were then incubated with $100 \mu \mathrm{l}$ 1X Apollo solution (Sigma-Aldrich; Merck KGaA) for $30 \mathrm{~min}$ at $37^{\circ} \mathrm{C}$ in the dark. Subsequently, the cells were incubated with $100 \mu 1$ 1X DAPI solution (Sigma-Aldrich; Merck KGaA) at room temperature for $30 \mathrm{~min}$ and washed with $100 \mu 1$ PBS prior to observation under a fluorescence microscope (Olympus Corporation).

Analysis of glucose uptake capacity. Glucose uptake was measured using a glucose uptake colorimetric assay kit (cat. no. ab136955; Abcam) according to the manufacturer's instructions. Briefly, the cells $(5,000$ cells/well) were serum-starved overnight in a 96-well plate with DMEM, and were then cultured with Krebs-Ringer-Phosphate-HEPES buffer (20 mM HEPES, $5 \mathrm{mM} \mathrm{KH}_{2} \mathrm{PO}_{4}, 1 \mathrm{mM} \mathrm{MgSO}{ }_{4}, 1 \mathrm{mM} \mathrm{CaCl}{ }_{2}, 136 \mathrm{mM} \mathrm{NaCl}$, $4.7 \mathrm{mM} \mathrm{KCl}$; $\mathrm{pH} 7.4$ ) containing $2 \%$ bovine serum albumin (Sigma-Aldrich; Merck KGaA) at room temperature for $40 \mathrm{~min}$. The cells were incubated in normal or high-glucose DMEM, and were treated with $10 \mu 1$ 2-deoxy-glucose (2-DG; Abcam) for $20 \mathrm{~min}$ at $37^{\circ} \mathrm{C}$. The concentration of high-glucose DMEM was $25 \mathrm{mM}$. The substrate oxidation reaction was measured at a wavelength of $412 \mathrm{~nm}$ with a microplate absorbance reader (Thermo Fisher Scientific, Inc.), and the data were organized by Microsoft Excel (Microsoft Office 2003).

Measurement of lactic acid formation. The levels of lactic acid were determined using a lactate assay kit (BioVision, Inc.). Cells $\left(5 \times 10^{6} /\right.$ well) were incubated with the reaction mixture in a 96-well plate at room temperature in the dark for $30 \mathrm{~min}$. The lactate level was examined using a microplate reader (Thermo Fisher Scientific, Inc.) at a wavelength of $450 \mathrm{~nm}$ before analysis by Microsoft Excel.

Determination of extracellular acidification rate $(E C A R)$ and oxygen consumption rate (OCR). The ECAR and OCR were determined every $7 \mathrm{~min}$ for 77 min using a Seahorse XFe96 analyzer (Seahorse Bioscience; Agilent Technologies, Inc.). Cells $\left(1 \times 10^{4} /\right.$ well $)$ were seeded in Seahorse XFe96 plates. After measuring the basal ECAR, the cells were incubated with $10 \mathrm{mM}$ glucose/well to test the capacity of glycolysis, followed by the addition of $1 \mu \mathrm{M}$ oligomycin for inhibition of oxidative phosphorylation to inspect the maximum glycolytic ability of the cells. Finally, the glycolysis inhibitor 2-DG (50 mM) was added to determine acid production by non-glycolytic pathways. All reagents were added at $0 \mathrm{~min}$ and the incubation temperature was maintained at $38.5^{\circ} \mathrm{C}$. Cells were detected every $7 \mathrm{~min}$ following continuous administration of $10 \mathrm{mM}$ glucose and inhibitors ( $1 \mu \mathrm{M}$ oligomycin and $50 \mathrm{mM}$ 2-DG). For the OCR examination, the basal OCR was first evaluated, after which the oxygen consumption for ATP synthesis was assessed after exposure to $2 \mu \mathrm{M}$ oligo, an ATP synthase inhibitor. The maximum oxygen consumption capacity of the cells was assessed after cells were given $2 \mu \mathrm{M}$ mitochondrial uncoupler (FCCP), and the cells were then treated with mitochondrial respiratory chain inhibitors antimycin $\mathrm{A}(0.5 \mu \mathrm{M})$ and oligomycin to prevent oxygen consumption by the mitochondria. All reagents were added at $0 \mathrm{~min}$ and the incubation temperature was maintained at $38.5^{\circ} \mathrm{C}$. Cells were measured every 7 min following 
Table I. Primer sequences of HK2 and GAPDH.

\begin{tabular}{lc}
\hline Name & \multicolumn{1}{c}{ Primer sequence } \\
\hline HK2 & F: 5'-AATGCTTCCATCTTATGCCCC-3' \\
& R:5'-CCACGAACACCAGGTTCAGG-3' \\
GAPDH & F: 5'-TCTTGTGCAGTGCCAGCCT-3' \\
& R: 5'-TGAGGTCAATGAAGGGGTCG-3'
\end{tabular}

F, forward; R, reverse; HK2, hexokinase-2.

continuous administration of 2.0 oligomycin, 2.0 FCCP and $0.5 \mu \mathrm{M}$ antimycin A. All reagents in this experiment were purchased from Sigma-Aldrich (Merck KGaA).

Reverse transcription-quantitative PCR (RT-qPCR). Total RNA was extracted from the cells using TRIzol ${ }^{\circledR}$ (Invitrogen; Thermo Fisher Scientific, Inc.) according to the manufacturer's protocol. After quantification using a spectrophotometer (Shimadzu Corporation), RNA samples were reverse transcribed into cDNA using a universal cDNA synthesis kit (Toyobo Life Science). The RT conditions were as follows: $37^{\circ} \mathrm{C}$ for $15 \mathrm{~min}, 85^{\circ} \mathrm{C}$ for $5 \mathrm{sec}$ and $4^{\circ} \mathrm{C}$. A SYBR Green PCR kit (Toyobo Life Science) was employed to perform RT-qPCR on the cDNA, and mRNA expression levels were detected using a PRISM 7300 real-time PCR system (Applied Biosystems; Thermo Fisher Scientific, Inc.). Samples underwent predenaturation at $95^{\circ} \mathrm{C}$ for $5 \mathrm{~min}$, followed by 40 cycles consisting of a 10 -sec denaturing step at $95^{\circ} \mathrm{C}$, a 10 -sec annealing step at $60^{\circ} \mathrm{C}$ and a 20 -sec extension step at $72^{\circ} \mathrm{C}$. The final extension was at $72^{\circ} \mathrm{C}$ for $10 \mathrm{~min}$. The reference gene of mRNA was set as GAPDH. Results were analyzed using the $2^{-\Delta \Delta \mathrm{Cq}}$ method (16) and the primers for GAPDH and HK2 are shown in Table I.

Western blotting. Cells were lysed with RIPA lysis buffer (Beyotime Institute of Biotechnology) to obtain the protein samples. The protein concentration was measured using a BCA kit (Pierce; Thermo Fisher Scientific, Inc.). Proteins (100 $\mu \mathrm{g} /$ well) were separated by $8 \%$ SDS-PAGE and transferred onto PVDF membranes. Subsequently, the membranes were blocked in $5 \%$ non-fat dry milk at room temperature for $1 \mathrm{~h}$, followed by incubation with primary antibodies against rabbit-derived GAPDH (1:10,000; cat. no. ab181602), HK2 (1:1,000; cat. no. ab209847), AKT (1:10,000; cat. no. ab179463) and phosphorylated (p)-AKT (1:1,000; cat. no. ab38449) (all Abcam) overnight at $4^{\circ} \mathrm{C}$. The membranes were then washed with $0.1 \%$ TBS-Tween-20 (TBST) and incubated with a goat anti-rabbit $\mathrm{IgG}$ secondary antibody (1:5,000; cat. no. CW0103; Beijing ComWin Biotech Co., Ltd.) at room temperature for $2 \mathrm{~h}$. After washing with TBST three times, blots were visualized in a Gel imaging system (GE Healthcare) using an Efficient chemiluminescence kit (Proandy). ImageJ software (version1 46; National Institutes of Health) was used to analyze the gray level of blots.

Statistical analysis. Statistical analysis was conducted using GraphPad Prism 7.0 software (GraphPad Software, Inc.). Data are expressed as the mean \pm SD $(n=3)$. Student's unpaired t-test was employed to compare between two groups. Comparisons among multiple groups were analyzed by one-way ANOVA followed by Dunnett's multiple comparison test. $\mathrm{P}<0.05$ was considered to indicate a statistically significant difference.

\section{Results}

GA suppresses the Warburg effect and proliferation in $L C$ cells and hinders activation of the PI3K/AKT pathway. The LC cell line HepG2 was exposed to different concentrations of GA $(0,25,50,75,100$ and $125 \mu \mathrm{g} / \mathrm{ml})$ for $24 \mathrm{~h}$ and incubated with CCK-8 solution to measure cell viability. It was revealed that the viability of HepG2 cells decreased with the increase in GA concentration, and the inhibitory effect of GA on cell viability was $\sim 50 \%$ when the concentration of GA was $100 \mu \mathrm{g} / \mathrm{ml}$ (Fig. 1A). GA at the concentration of $100 \mu \mathrm{g} / \mathrm{ml}$ exerted $\sim 50 \%$ inhibitory effect. Therefore, the concentration of GA at $100 \mu \mathrm{g} / \mathrm{ml}$ was selected for subsequent experiments. Subsequently, the CCK-8 assay was utilized to determine LC cell proliferation after HepG2 cells were exposed to $100 \mu \mathrm{g} / \mathrm{ml}$ GA. The results demonstrated that GA treatment significantly inhibited LC cell proliferation compared with that in the control group (Fig. 1A; GA vs. control group; $\mathrm{P}<0.05$ ), and the results of EdU staining corroborated this finding, in that a lower percentage of EdU-positive cells was detected in the GA group compared with the percentage detected in the control group (Fig. 1B; $\mathrm{P}<0.01$ ). These findings demonstrated the ability of GA to suppress cell proliferation. To investigate whether GA affects cell metabolism, glucose uptake capacity and lactic acid content were assessed in HepG2 cells; the results revealed that the glucose uptake capacity (Fig. 1C) and lactic acid content (Fig. 1D) of cells in the GA group were significantly lower than those in the control group $(\mathrm{P}<0.01)$.

Evaluation of the ECAR in glycolytic flux and the OCR in mitochondrial respiration demonstrated that LC cells in the GA group had an elevated OCR (Fig. 1F; GA vs. control group; $\mathrm{P}<0.01$ ) and a suppressed ECAR (Fig. 1E; GA vs. control group; $\mathrm{P}<0.01)$, indicating the suppressive role of $\mathrm{GA}$ on the Warburg effect. RT-qPCR (Fig. 1G; GA vs. control group) and western blotting (Fig. 1H; GA vs. control group) revealed that the expression levels of HK2 were diminished in HepG2 cells following GA exposure, illustrating that GA may hamper HK2 expression $(\mathrm{P}<0.01)$.

To further study the mechanism underlying the effects of GA on LC, the phosphorylation level of AKT in the PI3K/AKT signaling pathway was measured by western blotting. The results revealed that the phosphorylation level of AKT was decreased in the cells of the GA group compared with that in the control group (Fig. 1I; P<0.01), indicating that GA may interfere with the PI3K/AKT signaling pathway. Taken together, these observations suggested that GA may hinder the Warburg effect, cell proliferation and HK2 expression in LC cells. In addition, GA could block the PI3K/AKT pathway in LC cells.

HK2 facilitates LC cell proliferation and the Warburg effect. Considering the suppressive effects of GA on proliferation and HK2 expression in LC cells, it may be hypothesized that HK2 serves a possible regulatory role in the proliferation of LC cells. si-HK2 was transfected into HepG2 cells, and RT-qPCR (Fig. 2A) and western blotting (Fig. 2B) were 

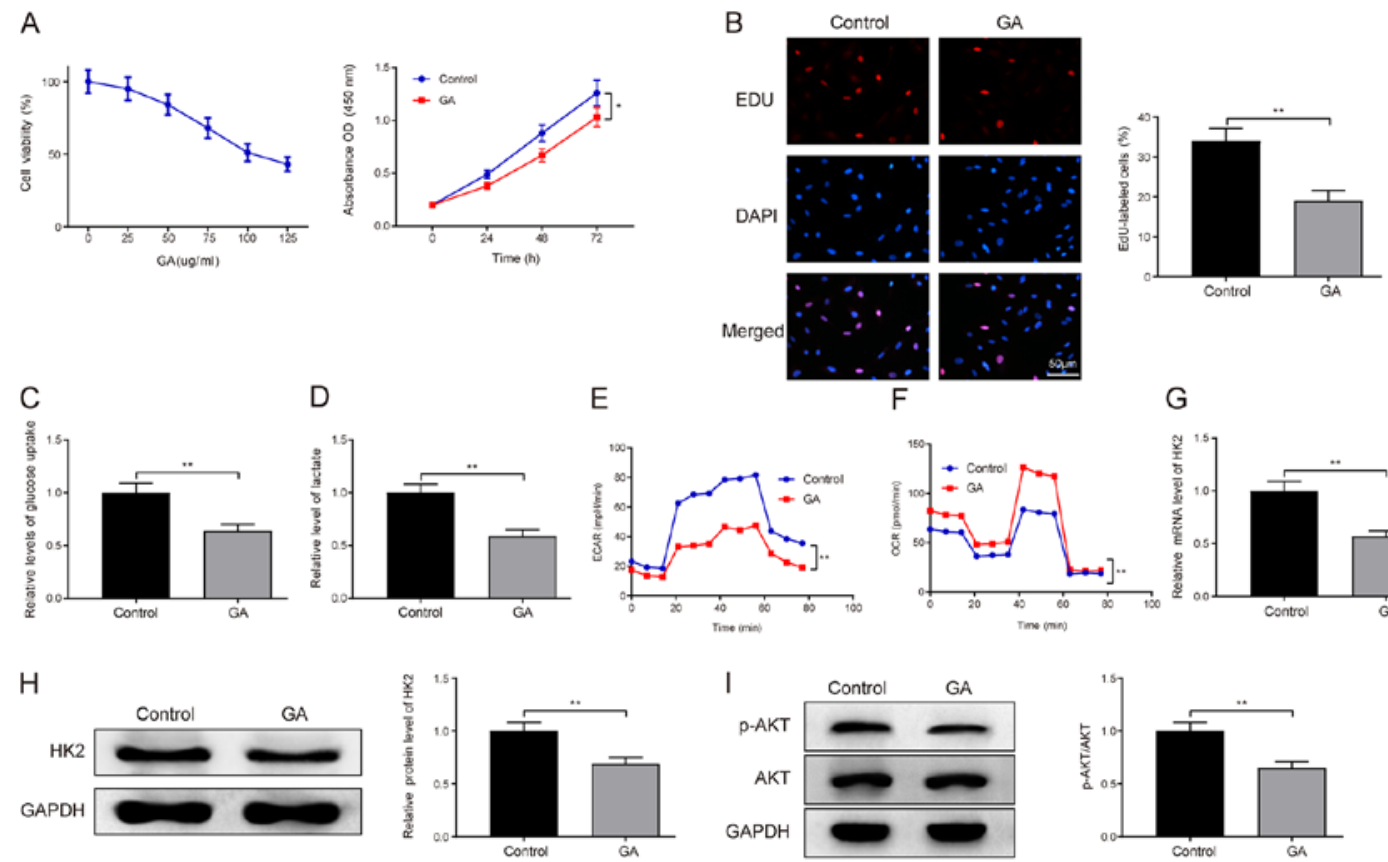

G
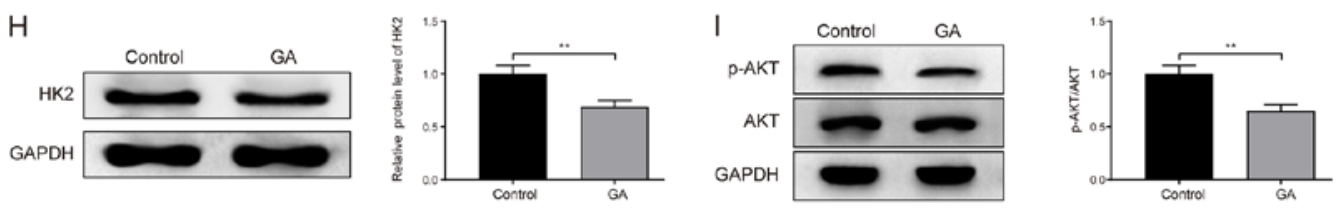

Figure 1. GA impedes LC cell proliferation and the Warburg effect, and blocks the PI3K/AKT pathway. The LC HepG2 cell line was treated with different concentrations of GA $(0,25,50,75,100$ and $125 \mu \mathrm{g} / \mathrm{ml})$ for $24 \mathrm{~h}$, followed by analysis of cell viability using the CCK-8 assay. Subsequently, $100 \mu \mathrm{g} / \mathrm{ml} \mathrm{GA}$ was chosen for the following experiments. (A) CCK-8 assay and (B) EdU staining were used to assess cell proliferation. (C) A glucose uptake colorimetric assay kit was used to measure glucose uptake capacity and (D) a lactate assay kit was used to assess lactic acid content. A Seahorse XFe96 analyzer was used to detect (E) ECAR and (F) OCR. (G) mRNA and (H) protein expression levels of HK2, and (I) the phosphorylation level of AKT, were analyzed by reverse transcription-quantitative PCR and western blotting. Data are presented as the mean \pm standard deviation. ${ }^{*} \mathrm{P}<0.05,{ }^{* * *} \mathrm{P}<0.01$. LC, liver cancer; CCK-8, Cell Counting Kit-8; ECAR, extracellular acidification rate; OCR, oxygen consumption rate; HK2, hexokinase-2; GA, glycyrrhizin; p-AKT, phosphorylated-AKT.

A

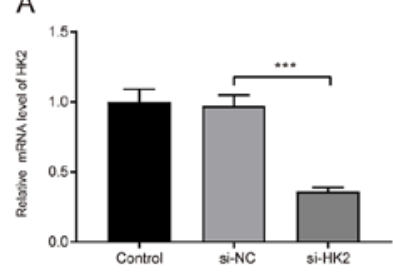

B
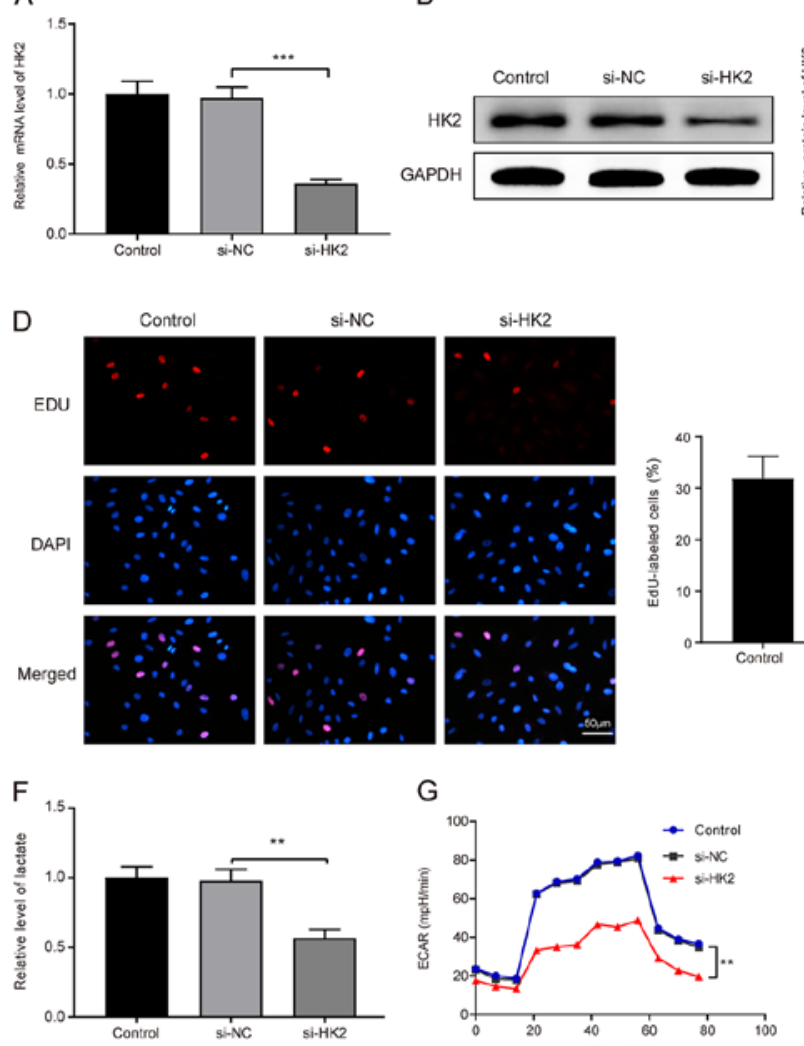

si-NC
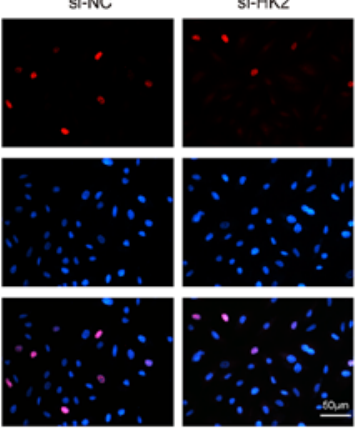

G

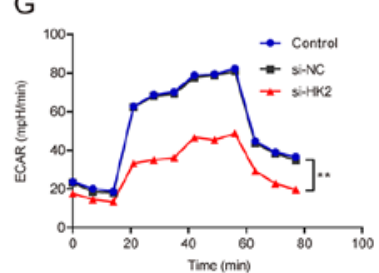

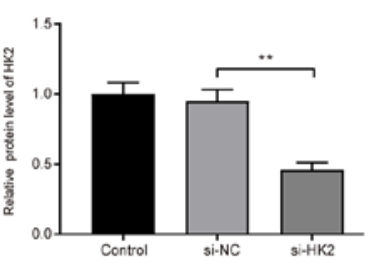

C

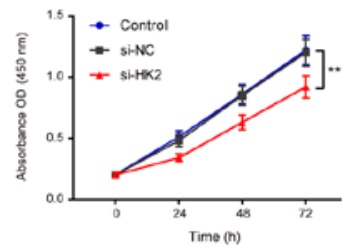

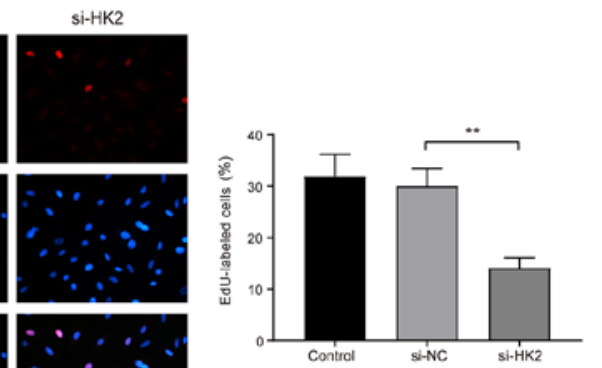

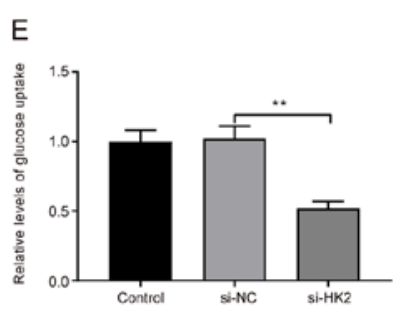

$\mathrm{H}$

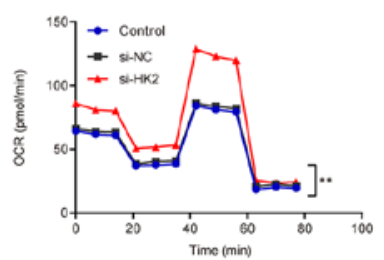

Figure 2. Liver cancer cell proliferation and the Warburg effect may be promoted by HK2. Transfection of HepG2 cells with si-HK2 was performed. The expression levels of HK2 were detected by (A) reverse transcription-quantitative PCR and (B) western blotting. Cell proliferation was assessed by (C) Cell Counting Kit-8 assay and (D) EdU staining. (E) A glucose uptake colorimetric assay kit was used to evaluate glucose uptake capacity and (F) a lactate assay kit was utilized to detect lactic acid content. (G) ECAR and (H) OCR were assessed using the Seahorse XFe96 analyzer. Data are presented as the mean \pm standard deviation. ${ }^{* * *} \mathrm{P}<0.01,{ }^{* * * *} \mathrm{P}<0.001$. ECAR, extracellular acidification rate; OCR, oxygen consumption rate; HK2, hexokinase-2; si, small interfering; NC, negative control. 


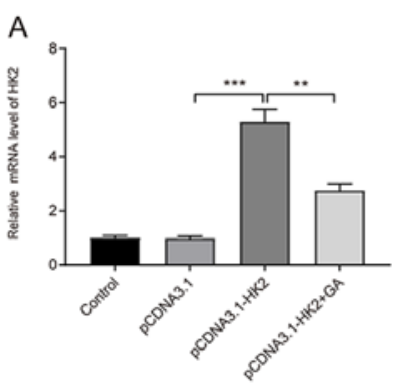

B
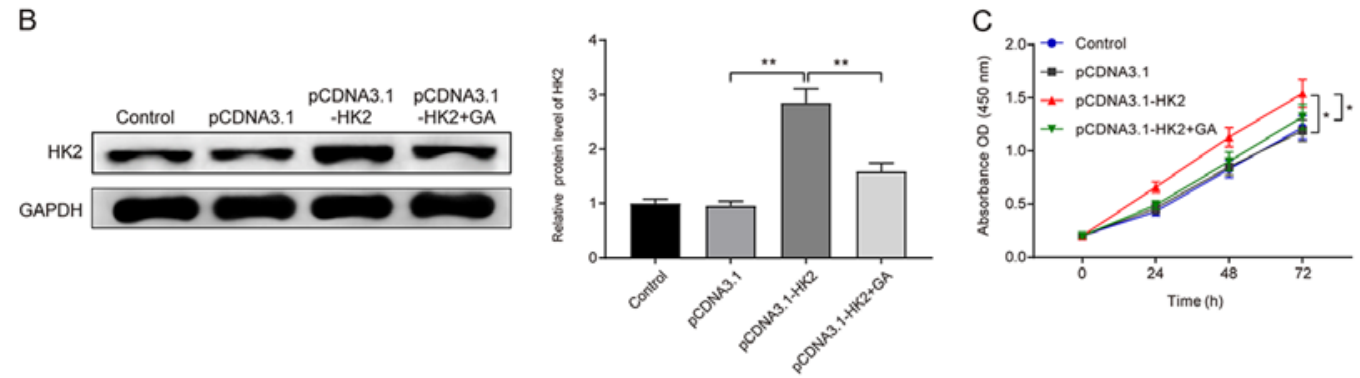

D
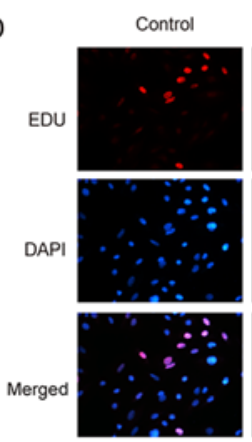

$\mathrm{F}$
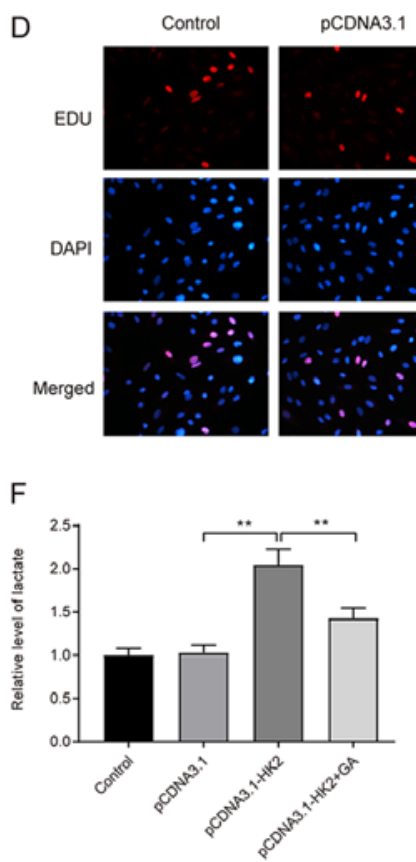
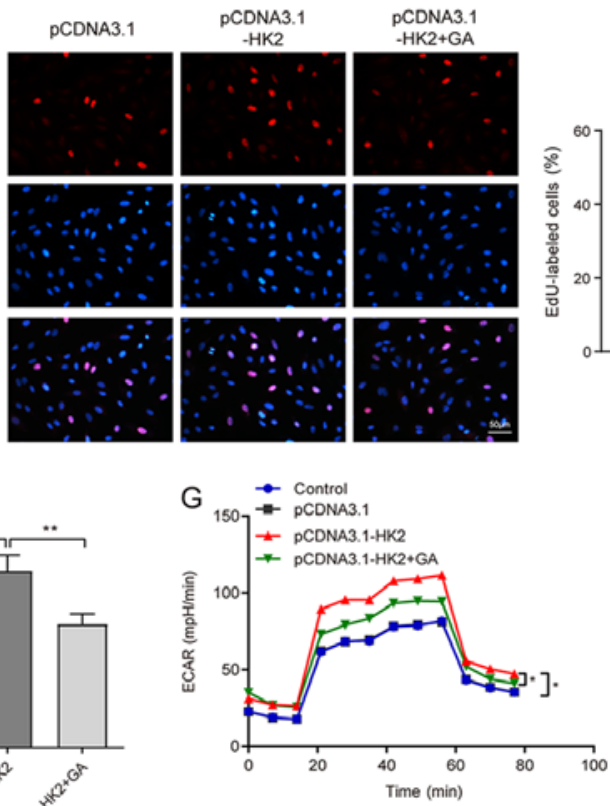

E
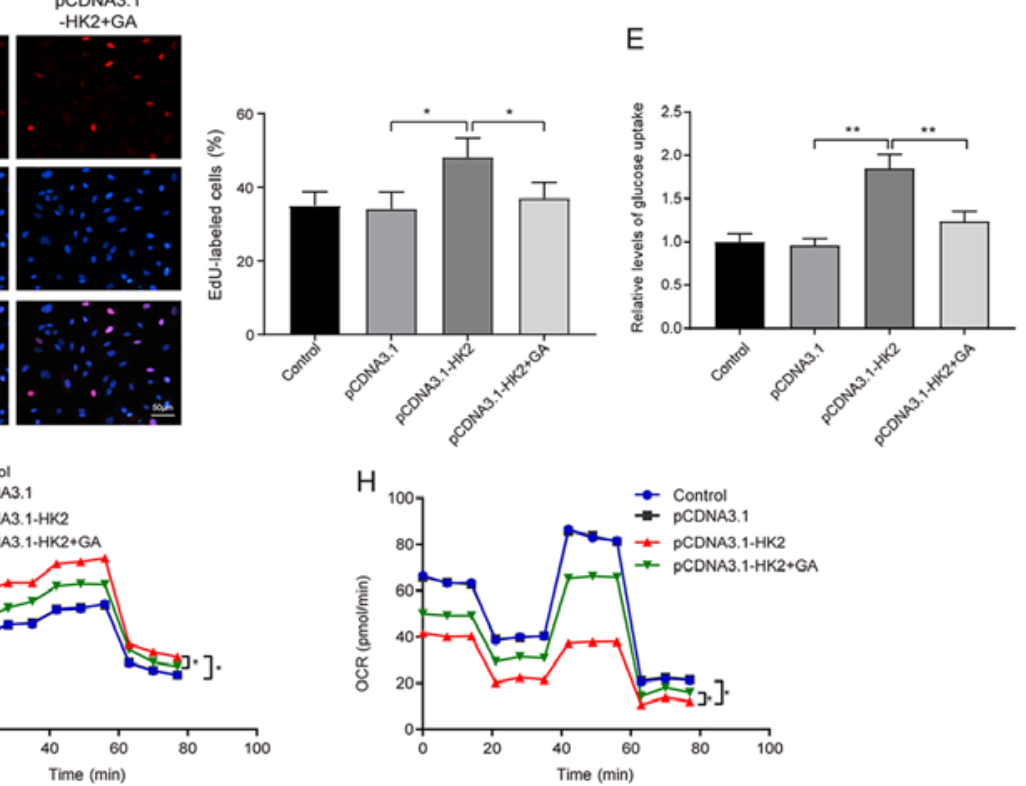

Figure 3. GA inhibits HK2 expression to suppress proliferation and the Warburg effect in liver cancer cells. Cells were transfected with pCDNA3.1-HK2 followed by GA treatment. (A) Reverse transcription-quantitative PCR and (B) western blotting were employed to examine the mRNA and protein expression levels of HK2. (C) A Cell Counting Kit-8 assay and (D) EdU staining were performed to assess cell proliferation. (E) Glucose uptake capacity, (F) lactic acid content, (G) ECAR and (H) OCR were analyzed. Data are presented as the mean \pm standard deviation. ${ }^{*} \mathrm{P}<0.05$, ${ }^{* *} \mathrm{P}<0.01,{ }^{* * * *} \mathrm{P}<0.001$. ECAR, extracellular acidification rate; OCR, oxygen consumption rate; HK2, hexokinase-2; GA, glycyrrhizin.

utilized to examine the transfection efficiency; HK2 was found to be successfully silenced in HepG2 cells $(\mathrm{P}<0.001$ and $\mathrm{P}<0.01)$. A CCK -8 assay revealed that transfection with si-HK2 suppressed LC cell proliferation (Fig. 2C; si-HK2 vs. si-NC group; $\mathrm{P}<0.01)$. In addition, EdU staining revealed that there was a lower percentage of EdU-positive cells in the si-HK2 group compared with in the si-NC group (Fig. 2D; $\mathrm{P}<0.01)$. These data suggested that $\mathrm{HK} 2$ may promote LC cell proliferation.

Glucose uptake capacity (Fig. 2E) and lactic acid content (Fig. 2F) were inhibited in the si-HK2 group compared with those in the si-NC group $(\mathrm{P}<0.01)$. Additionally, exposure to si-HK2 enhanced mitochondrial respiration (Fig. 2H; si-HK2 vs. si-NC group; $\mathrm{P}<0.01$ ) and lessened glycolytic flux (Fig. 2G; si-HK2 vs. si-NC group; $\mathrm{P}<0.01)$, which confirms the promotive role of HK2 in the Warburg effect. The aforementioned indicators remained unchanged in the si-NC group and the control group. Collectively, HK2 may promote the proliferation of LC cells and facilitate the Warburg effect to regulate cell metabolism.

HK2 suppresses the effect of GA on LC cell proliferation and the Warburg effect. Following transfection with pCDNA3.1-HK2,
HepG2 cells were treated with GA. RT-qPCR (Fig. 3A) and western blotting (Fig. 3B) analyses revealed that transfection with pCDNA3.1-HK2 significantly increased the mRNA and protein expression levels of HK2 (pDNA3.1-HK2 group vs. pCDNA3.1 group; $\mathrm{P}<0.001$ and $\mathrm{P}<0.01)$, whereas HK2 expression was suppressed following GA treatment (pCDNA3.1-HK2+GA group vs.pDNA3.1-HK2 group; $\mathrm{P}<0.01$ ). A CCK-8 assay (Fig. 3C) and EdU staining (Fig. 3D) illustrated that transfection with pCDNA3.1-HK2 significantly stimulated LC cell proliferation (pDNA3.1-HK2 vs. pCDNA3.1 group; $\mathrm{P}<0.05$ and $\mathrm{P}<0.05$ ); however, following GA exposure, cell proliferation was inhibited (pCDNA3.1-HK2 + GA group vs. pDNA3.1-HK2 group; $\mathrm{P}<0.05$ and $\mathrm{P}<0.05$ ), demonstrating that GA can suppress LC cell proliferation by HK2 reduction.

Progressive increases in glucose uptake capacity (Fig. 3E) and lactic acid content (Fig. 3F) were detected in the pCDNA3.1-HK2 group compared with those in the pCDNA3.1 and pCDNA3.1-HK2+GA groups $(\mathrm{P}<0.01)$. In addition, ECAR and OCR analyses demonstrated that cells in the pCDNA3.1-HK2 group had a higher ECAR (Fig. 3G; $\mathrm{P}<0.05$ ) and a lower OCR (Fig. $3 \mathrm{H} ; \mathrm{P}<0.05)$ compared with those in the pCDNA3.1 group. However, compared with the pCDNA3.1-HK2 group, the pCDNA3.1-HK2 + GA group 

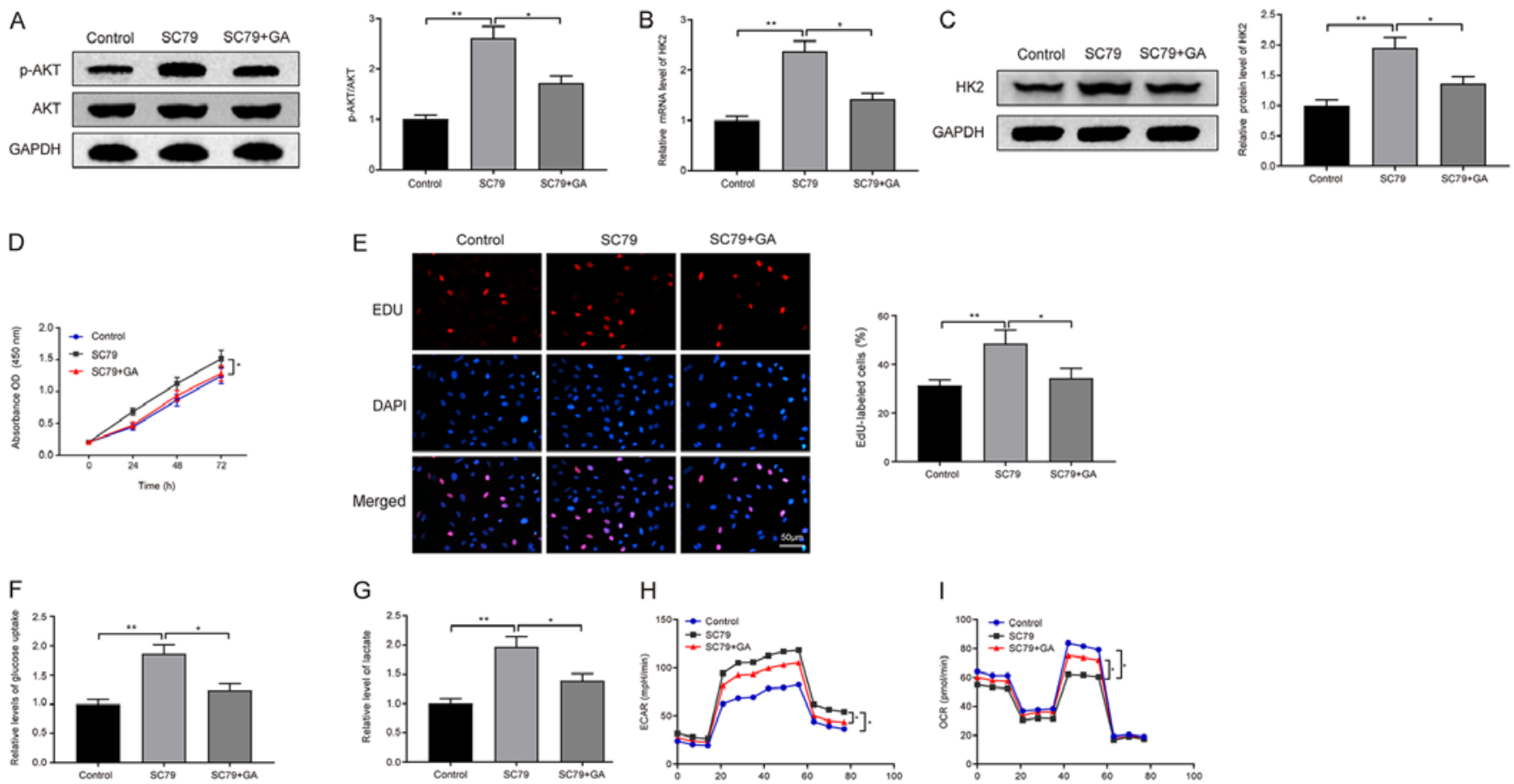

G

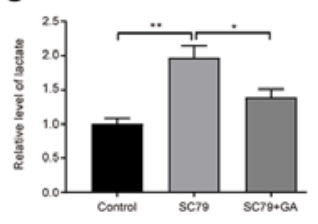

$\mathrm{H}$

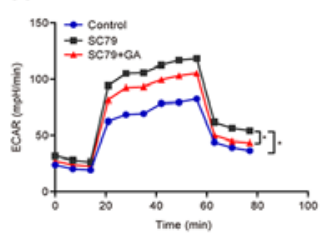

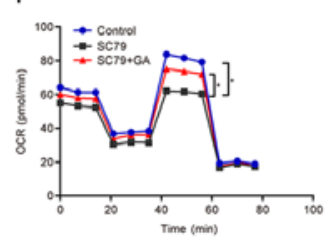

Figure 4. Inhibition of liver cancer cell proliferation and the Warburg effect by GA is associated with HK2 suppression through the PI3K/AKT signaling pathway. After HepG2 cells were treated with the AKT agonist and GA, the (A) phosphorylation level of AKT and (B and C) expression of HK2 were measured by reverse transcription-quantitative PCR and western blotting. (D) The Cell Counting Kit-8 assay and (E) EdU staining were employed to assess cell proliferation. (F) Glucose uptake capacity, (G) lactic acid content, (H) ECAR and (I) OCR were analyzed. Data are presented as the mean \pm standard deviation. " $\mathrm{P}<0.05$, ${ }^{* *} \mathrm{P}<0.01$. ECAR, extracellular acidification rate; OCR, oxygen consumption rate; HK2, hexokinase-2; GA, glycyrrhizin; p-AKT, phosphorylated-AKT.

exhibited an elevated OCR and a decreased ECAR $(\mathrm{P}<0.05)$. Taken together, these findings suggested that GA may suppress the Warburg effect and proliferation of LC cells through the suppression of HK2.

GA mediates its effects on HK2 through the PI3K/AKT pathway to inhibit proliferation and the Warburg effect in $L C$ cells. HepG2 cells were incubated with the AKT agonist SC79 and GA to investigate the specific mechanism underlying the effects of GA on LC. Western blotting results on the phosphorylation level of AKT revealed that treatment with the AKT agonist SC79 increased the phosphorylation level of AKT (SC79 vs. control group; $\mathrm{P}<0.01$ ), whereas the phosphorylation of AKT was decreased following exposure to GA (Fig. 4A; SC79 + GA vs. SC79 group; $\mathrm{P}<0.05)$. In addition, significant increases in the mRNA and protein expression levels of HK2 were detected in the SC79 group compared with those in the control and SC79 + GA groups (Fig. 4B and C; $\mathrm{P}<0.01$ and $\mathrm{P}<0.05$ ), indicating that the $\mathrm{PI} 3 \mathrm{~K} / \mathrm{AKT}$ pathway was associated with the effects of GA on HK2 expression. Additionally, SC79 promoted LC cell proliferation (SC79 vs. control group), whereas this effect was reversed by GA (Fig. 4D and E; SC79 + GA vs. SC79 group; $\mathrm{P}<0.05$ and $\mathrm{P}<0.01)$.

Elevated glucose uptake capacity (Fig. 4F) and lactic acid content (Fig. 4G) were detected in the SC79 group compared with those in the control and SC79 + GA groups $(\mathrm{P}<0.01$ and $\mathrm{P}<0.05)$. Furthermore, exposure to SC79 elevated the ECAR and suppressed the OCR (SC79 vs. control group), whereas treatment with a combination of SC79 and GA reversed these trends (Fig. 4H and I; SC79 + GA vs. SC79 group; P<0.05).

\section{Discussion}

At present, therapeutic options for the treatment of LC are principally composed of radiological intervention, tumor resection, liver transplant and chemotherapy, all of which are limited to patients with early stage disease (17). Cancer cell metabolism has emerged as a field of biology that provides novel access to cancer treatments (18). Therefore, a better understanding of the molecular basis of LC formation and cell metabolism is essential for improvements in prognosis. In the present study, HepG2 cells were exposed to GA to determine the potential functions and possible mechanism of GA on cell proliferation and the Warburg effect. The present findings demonstrated that GA could suppress cell proliferation and metabolism through suppressing HK2 expression via blockade of the PI3K/AKT signaling pathway.

The Warburg effect confers a growth advantage on cancer cells and HK2 has a vital role in this process $(9,19)$. High expression levels of HK2 have been observed in LC cells $(20,21)$. HK2 was here revealed to accelerate LC cell proliferation and glycolytic metabolism, as evidenced by an increase in mitochondrial respiration, and decreases in glucose uptake capacity, lactic acid content, glycolytic flux and proliferation in HepG2 cells following transfection with si-HK2. The present findings are supported by a study by Zhang et al (22), which reported that the Polygonatum cyrtonema lectin suppressed tumor cell glycolysis in PC3 cells by silencing HK2 and combining with epidermal growth factor receptor. As the extensively studied sweet-tasting constituent of licorice, GA is a natural triterpenoid saponin glycoside 
obtained from licorice roots (23). GA was previously shown to suppress cell proliferation in human leukemia and markedly reduced the growth of leukemia cells via inhibition of the AKT/mTOR/STAT3 signaling pathway (24). It has also been reported that GA may exert protective effects against high glucose-induced cell proliferation and oxidative stress in NRK-52E cells (25). A significant finding of the present study was the observation that GA was a crucial mediator of LC cell proliferation and the Warburg effect, and that it affected the expression levels of HK2. These results prompted analysis of whether GA elicits a suppressive effect on LC through regulation of HK2. HepG2 cells were treated with pCDNA3.1-HK2 and GA, and the findings of the CCK-8 assay, EdU staining, ECAR, OCR, RT-qPCR and western blotting demonstrated that overexpression of HK2 elevated cell proliferation, glucose uptake capacity, lactic acid content and glycolytic flux, and suppressed mitochondrial respiration. Conversely, following exposure to GA, these effects were reversed. Collectively, these data confirmed that GA may suppress proliferation and the Warburg effect in LC cells through inhibition of HK2.

Following the confirmation that GA hampered LC progression, the aim of the present study shifted to determining the specific signaling pathway implicated in this protection. AKT is one of the primary downstream targets of the PI3K signaling pathway and a key cell survival factor (26). The PI3K/AKT signaling pathway, which is involved in the regulation of cell proliferation and the Warburg effect, has been documented to serve a major role not only in tumor development but also in the potential response of tumors to cancer treatment (27-29). To assess the effect of the PI3K/AKT signaling pathway on LC cells, the $\mathrm{PI} 3 \mathrm{~K} / \mathrm{AKT}$ signaling pathway was activated, after which the LC cells were treated with GA to observe its effect. These results revealed that activation of the PI3K/AKT signaling upregulated HK2 expression and facilitated LC cell proliferation and the Warburg effect, whereas GA could reverse the effects exerted by the PI3K/AKT pathway.

In conclusion, the findings of the present study revealed that GA exerted an inhibitory effect on the Warburg effect and proliferation of LC cells. GA was able to silence HK2 through the PI3K/AKT pathway, in order to inhibit LC cell proliferation and glycolytic metabolism. The specific role of GA in the Warburg effect in LC determined in the present study may improve the theoretical understanding of glucose metabolism reprogramming in human LC and could indicate potential therapeutic targets to treat the disease. Nonetheless, these results must be interpreted with caution and further investigation is required to focus on the issues emerging from the present study.

\section{Acknowledgements}

Not applicable.

\section{Funding}

This research was funded by the Foundation of Hunan Provincial Health Commission (grant no. 20200179) and the Changsha Bureau of Science and Technology Planning Foundation (grant no. kq1706032).

\section{Availability of data and materials}

The datasets used and/or analyzed during the current study are available from the corresponding author on reasonable request.

\section{Authors' contributions}

ZS and CP conceptualized and supervised the study, designed the experiments and analyzed the data. ZS, ZT and WY performed the experiments and wrote the manuscript. ZS and WY provided critical materials. ZS and CP authenticated all the raw data. All authors read and approved the final manuscript.

\section{Ethics approval and consent to participate}

Not applicable.

\section{Patient consent for publication}

Not applicable.

\section{Competing interests}

The authors declare that they have no competing interests.

\section{References}

1. Anwanwan D, Singh SK, Singh S, Saikam V and Singh R: Challenges in liver cancer and possible treatment approaches. Biochim Biophys Acta Rev Cancer 1873: 188314, 2020.

2. Salvatore M, Jeon J and Meza R: Changing trends in liver cancer incidence by race/ethnicity and sex in the US: 1992-2016. Cancer Causes Control 30: 1377-1388, 2019.

3. Pittala S, Krelin Y and Shoshan-Barmatz V: Targeting Liver Cancer and Associated Pathologies in Mice with a Mitochondrial VDAC1-Based Peptide. Neoplasia 20: 594-609, 2018.

4. Tran KT, McMenamin UC, Coleman HG, Cardwell CR, Murchie P, Iversen L, Lee AJ and Thrift AP: Statin use and risk of liver cancer: Evidence from two population-based studies. Int J Cancer 146: 1250-1260, 2020.

5. Guo Z, Zhou Y, Yang J and Shao X: Dendrobium candidum extract inhibits proliferation and induces apoptosis of liver cancer cells by inactivating Wnt/ $\beta$-catenin signaling pathway. Biomed Pharmacother 110: 371-379, 2019.

6. Nie H, Li J, Yang XM, Cao QZ, Feng MX, Xue F, Wei L, Qin W, Gu J, Xia Q, et al: Mineralocorticoid receptor suppresses cancer progression and the Warburg effect by modulating the miR-338-3p-PKLR axis in hepatocellular carcinoma. Hepatology 62: 1145-1159, 2015.

7. Xu F, Yan JJ, Gan Y, Chang Y, Wang HL, He XX and Zhao Q: miR-885-5p Negatively Regulates Warburg Effect by Silencing Hexokinase 2 in Liver Cancer. Mol Ther Nucleic Acids 18: 308-319, 2019.

8. Zhou Y, Zheng X, Lu J, Chen W, Li X and Zhao L: Ginsenoside 20(S)-Rg3 Inhibits the Warburg Effect Via Modulating DNMT3A/miR-532-3p/HK2 Pathway in Ovarian Cancer Cells. Cell Physiol Biochem 45: 2548-2559, 2018.

9. Lin G, Wu Y, Cai F, Li Z, Su S, Wang J, Cao J and Ma L: Matrine Promotes Human Myeloid Leukemia Cells Apoptosis Through Warburg Effect Mediated by Hexokinase 2. Front Pharmacol 10: $1069,2019$.

10. Jin F, Wang Y, Zhu Y, Li S, Liu Y, Chen C, Wang X, Zen K and $\mathrm{Li} \mathrm{L}$ : The miR-125a/HK2 axis regulates cancer cell energy metabolism reprogramming in hepatocellular carcinoma. Sci Rep 7: 3089, 2017.

11. Kong ZL, Kuo HP, Johnson A, Wu LC and Chang KLB: Curcumin-Loaded Mesoporous Silica Nanoparticles Markedly Enhanced Cytotoxicity in Hepatocellular Carcinoma Cells. Int J Mol Sci 20: 20, 2019. 
12. Zhang $P$, Wang Q, Lin Z, Yang P, Dou K and Zhang R: Berberine Inhibits Growth of Liver Cancer Cells by Suppressing Glutamine Uptake. OncoTargets Ther 12: 11751-11763, 2019.

13. Liang B, Guo XL, Jin J, Ma YC and Feng ZQ: Glycyrrhizic acid inhibits apoptosis and fibrosis in carbon-tetrachloride-induced rat liver injury. World J Gastroenterol 21: 5271-5280, 2015.

14. Cao ZY, Liu YZ, Li JM, Ruan YM, Yan WJ, Zhong SY, Zhang T, Liu LL, Wu R, Wang B, et al: Glycyrrhizic acid as an adjunctive treatment for depression through anti-inflammation: A randomized placebo-controlled clinical trial. J Affect Disord 265: 247-254, 2020.

15. Li JY, Cao HY, Liu P, Cheng GH and Sun MY: Glycyrrhizic acid in the treatment of liver diseases: Literature review. BioMed Res Int 2014: 872139, 2014.

16. Livak KJ and Schmittgen TD: Analysis of relative gene expression data using real-time quantitative PCR and the 2(-Delta Delta C(T)) Method. Methods 25: 402-408, 2001.

17. Lee NCW, Carella MA, Papa S and Bubici C: High Expression of Glycolytic Genes in Cirrhosis Correlates With the Risk of Developing Liver Cancer. Front Cell Dev Biol 6: 138, 2018.

18. Wang Z, Deng M, Liu Z and Wu S: Hypoxia-induced miR-210 promoter demethylation enhances proliferation, autophagy and angiogenesis of schwannoma cells. Oncol Rep 37: 3010-3018, 2017.

19. Guo W, Qiu Z, Wang Z, Wang Q, Tan N, Chen T, Chen Z, Huang S, $\mathrm{Gu} \mathrm{J}, \mathrm{Li}$ J, et al: miR-199a-5p is negatively associated with malignancies and regulates glycolysis and lactate production by targeting hexokinase 2 in liver cancer. Hepatology 62: 1132-1144, 2015.

20. Jiao L, Zhang HL, Li DD, Yang KL, Tang J, Li X, Ji J, Yu Y, Wu RY, Ravichandran S, et al: Regulation of glycolytic metabolism by autophagy in liver cancer involves selective autophagic degradation of HK2 (hexokinase 2). Autophagy 14: 671-684, 2018.

21. Zhuang X, Chen Y, Wu Z, Xu Q, Chen M, Shao M, Cao X, Zhou Y, Xie M, Shi Y, et al: Mitochondrial miR-181a-5p promotes glucose metabolism reprogramming in liver cancer by regulating the electron transport chain. Carcinogenesis 41 : $972-983,2020$
22. Zhang H, Du X, Sun TT, Wang CL, Li Y and Wu SZ: Lectin PCL inhibits the Warburg effect of PC 3 cells by combining with EGFR and inhibiting HK2. Oncol Rep 37: 1765-1771, 2017.

23. Khan R, Rehman MU, Khan AQ, Tahir M and Sultana S: Glycyrrhizic acid suppresses 1,2-dimethylhydrazine-induced colon tumorigenesis in Wistar rats: Alleviation of inflammatory, proliferation, angiogenic, and apoptotic markers. Environ Toxicol 33: 1272-1283, 2018

24. He SQ, Gao M, Fu YF and Zhang YN: Glycyrrhizic acid inhibits leukemia cell growth and migration via blocking AKT/mTOR/STAT3 signaling. Int J Clin Exp Pathol 8: 5175-5181, 2015.

25. Hou S, Zheng F, Li Y, Gao L and Zhang J: The protective effect of glycyrrhizic acid on renal tubular epithelial cell injury induced by high glucose. Int J Mol Sci 15: 15026-15043, 2014.

26. Liu W, Jing ZT, Xue CR, Wu SX, Chen WN, Lin XJ and Lin X: PI3K/AKT inhibitors aggravate death receptor-mediated hepatocyte apoptosis and liver injury. Toxicol Appl Pharmacol 381: 114729,2019

27. Fresno Vara JA, Casado E, de Castro J, Cejas P, Belda-Iniesta C and González-Barón M: PI3K/Akt signalling pathway and cancer. Cancer Treat Rev 30: 193-204, 2004.

28. Hu F, He Z, Sun C and Rong D: Knockdown of GRHL2 inhibited proliferation and induced apoptosis of colorectal cancer by suppressing the PI3K/Akt pathway. Gene 700: 96-104, 2019.

29. Liu J, Pan C, Guo L, Wu M, Guo J, Peng S, Wu Q and Zuo Q: A new mechanism of trastuzumab resistance in gastric cancer: MACC1 promotes the Warburg effect via activation of the PI3K/AKT signaling pathway. J Hematol Oncol 9: 76, 2016.

This work is licensed under a Creative Commons Attribution-NonCommercial-NoDerivatives 4.0 International (CC BY-NC-ND 4.0) License. 